\title{
Commissioning the MMT ground- layer and laser tomography adaptive optics systems
}

N. Mark Milton, Michael Lloyd-Hart, Christoph Baranec, Thomas Stalcup, Keith Powell, et al.

N. Mark Milton, Michael Lloyd-Hart, Christoph Baranec, Thomas Stalcup, Keith Powell, Don McCarthy, Craig Kulesa, Keith Hege, "Commissioning the MMT ground-layer and laser tomography adaptive optics systems," Proc. SPIE 7015, Adaptive Optics Systems, 701522 (11 July 2008); doi: 10.1117/12.788541

Event: SPIE Astronomical Telescopes + Instrumentation, 2008, Marseille, France 


\title{
Commissioning of the MMT ground-layer and laser tomography adaptive optics systems
}

\author{
N. Mark Milton ${ }^{1}$, Michael Lloyd-Hart ${ }^{1}$, Christoph Baranec ${ }^{2}$, Thomas Stalcup ${ }^{1}$, Keith Powell ${ }^{1}$, \\ Don McCarthy ${ }^{1}$, Craig Kulesa ${ }^{1}$, and Keith Hege ${ }^{1}$ \\ ${ }^{1}$ Center for Astronomical Adaptive Optics, Steward Observatory, \\ 933 N. Cherry Ave., Tucson, Arizona 85721 \\ ${ }^{2}$ Caltech Optical Observatories, California Institute of Technology, \\ 1200 E. California Blvd., Pasadena, California 91125
}

\begin{abstract}
A multi-laser adaptive optics system, at the $6.5 \mathrm{~m}$ MMT telescope, has been undergoing commissioning in preparation for wide-field, partially corrected as well as narrow-field, diffraction limited science observations in the thermal and near infrared. After several delays due to bad weather, we have successfully closed the full high order ground-layer adaptive optics (GLAO) control loop for the first time in February 2008 using five Rayleigh laser guide stars and a single tilt star. Characterization and automated correction of static aberrations such as non-common path errors were addressed in May 2008. Calibration measurements in preparation for laser tomography adaptive optics (LTAO) operation are planned for the fall of 2008 along with the start of shared-risk GLAO science observations.

We present the results of GLAO observations with the PISCES imager, a $1-2.5 \mu \mathrm{m}$ camera with a field of view of 110 arc seconds. The status of the remaining GLAO commissioning work is also reviewed. Finally, we present plans for commissioning work to implement the LTAO operating mode of the system.
\end{abstract}

Keywords: adaptive optics, ground-layer adaptive optics, laser guide stars

\section{INTRODUCTION}

Until very recently, adaptive optics (AO) systems, in operation at large astronomical telescopes, have used a single guide star to measure the atmospheric induced aberrations that degrade the long exposure imaging performance of science observations. Whether using a natural star or artificial beacon, the highest level of image correction has been limited to a single science target. The quality of aberration correction, and hence the image quality, for nearby targets degrades quickly with increasing field angle from the primary science target.

With the availability of multiple guide stars, several additional modes of AO correction can be implemented to address the issue of the limited $\mathrm{AO}$ corrected field. In particular, ground-layer adaptive optics (GLAO), first suggested by Rigaut, ${ }^{1}$ provides a method for achieving uniform, partial correction over fields as large as 10 arc minutes and has received significant interest for use in wide field imaging and spectroscopic surveys. A groundlayer estimate of the aberrations present close to the telescope aperture, which are common to all beacons, is easily produced by averaging the reconstructed aberration measurements from multiple beacons. The ground layer aberration is corrected by a deformable mirror (DM) conjugate to the telescope pupil.

Numerous simulations and open-loop measurements have predicted that multiple-beacon GLAO can effectively and consistently improve the atmospheric seeing. ${ }^{1-7}$ GLAO was first demonstrated on-sky in closed-loop using three bright natural guide stars in a 1.5 arc minute diameter field of view with the Multi-Conjugate Adaptive Optics Demonstrator fielded at the VLT in early 2007.8,9

Single, low altitude Rayleigh laser guide star (LGS) systems at smaller telescopes such as at the $4.2 \mathrm{~m} \mathrm{WHT}^{10}$ and planned for the $4.1 \mathrm{~m} \mathrm{SOAR}^{11}$ telescope can also produce wavefront compensation over fields of 1.5 and 3 arc minutes respectively. However, a single beacon GLAO implementation will not be effective for larger apertures

Send correspondence to N. M. Milton, E-mail: nmilton@as.arizona.edu

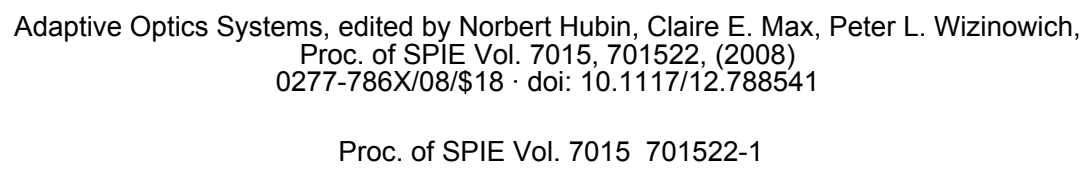


which suffer from stronger focal anisoplanatism effects that limit the accuracy of the ground-layer turbulence measurements. ${ }^{2}$ Multiple laser guide stars will be required too for GLAO implementations that achieve full sky coverage on the next generation of $30 \mathrm{~m}$ class telescopes.

The multi-laser guide star AO system on the $6.5 \mathrm{~m}$ MMT telescope, at Mt. Hopkins, Arizona, uses a constellation of five Rayleigh laser beacons projected in a regular pentagon with a diameter of $2 \operatorname{arc~minutes.}{ }^{5-7}$ The five reconstructed wavefronts, combined with tip-tilt information from a nearby natural star, have been successfully used in closed loop to correct for ground-layer aberrations using the MMT's adaptive secondary mirror (ASM). Image quality in the near IR is recorded by PISCES, an imaging camera with 110 arc second field of view.

After several delays due to bad weather, closed loop GLAO observations were performed in February 2008. Section 2 presents results from the February telescope run as well as from a subsequent run in May 2008 where initial tests of automated correction of static aberrations such as non-common path errors were successfully

carried out. Significant image improvement across the full field of PISCES was seen, but not yet at the anticipated resolution of 0.1 to 0.2 arc seconds in the $\mathrm{K}$ band projected by earlier analysis. ${ }^{6}$ System performance is limited for now because, except under conditions with almost no wind, it can be operated only at very low loop gain. This is because of a resonance at $2.3 \mathrm{~Hz}$ in the MMT's elevation drive which is presently amplified rather than suppressed by the drive servo. The resulting image motion, although it is very well sensed by the LGS system's natural guide star tip-tilt sensor, cannot be adequately corrected by the limited stroke of the ASM. This issue must be addressed in order for the LGS AO system to achieve the target performance.

The MMT laser adaptive optics system is currently being commissioned to support science observations in GLAO mode and laser tomographic adaptive optics (LTAO) mode, where multiple lasers are used to maximize on-axis Strehl. Section 3 describes the ongoing work to prepare for GLAO shared risk science. Two areas of immediate scientific application of GLAO are the study of galaxies at high redshift and the characterization of the initial mass function (IMF) of low mass and sub-stellar objects. We also discuss the LTAO commissioning work anticipated for the fall of 2008.

\section{GLAO OBSERVATIONS AND ANALYSIS}

\subsection{Instrument}

The MMT multi-laser guide star AO system consists of a laser beam projector, ${ }^{12}$ an adaptive secondary mirror, ${ }^{13,14}$ a Cassegrain mounted wavefront sensor ${ }^{5-7,15}$ (WFS), and a PC based real-time reconstructor. The beam projection optics project a constellation of five Rayleigh LGS from behind the telescope secondary mirror, on the sky in a regular pentagon with a diameter of 2 arc minutes and 25 watts of total power at $532 \mathrm{~nm}$.

The LGS WFS uses dynamically refocused optics ${ }^{16}$ and an electronically shuttered CCD to accumulate photon return over the range $20-29 \mathrm{~km}$. Hartmann patterns with 60 subapertures in a hexapolar geometry, one for each of the five LGS, are created by a prism array and captured on the LGS WFS CCD at $400 \mathrm{~Hz}$. An electron multiplying L3 CCD is used to obtain $400 \mathrm{~Hz}$ tip-tilt measurements from a natural star within the 2 arc minute LGS constellation. Finally, a traditional natural guide star (NGS) $12 \times 12$ Shack-Hartmann WFS is available for system calibration and automatic static aberration correction.

The Cassegrain mounted wavefront sensor is designed to accept all of the current suite of MMT $f / 15$ NGS AO science instruments including PISCES,${ }^{17}$ Clio, ${ }^{18}$ ARIES, ${ }^{19}$ and BLINC-MIRAC. ${ }^{20}$

The GLAO correction is calculated by reconstructor matrix multiplication in the PC based real-time computer from 300 LGS slope pairs, 60 pairs from each of the five laser beacons, as well as a pair of slopes from the fast tiptilt camera. The reconstructor is formed by first projecting each of the five sets of LGS WFS slope measurements onto an orthogonal basis of disk harmonic (DH) functions. ${ }^{21}$ These five LGS measurements are averaged, mode by mode, to produce an estimate of the ground-layer contribution to atmospheric seeing. Finally, the GLAO modal estimate is converted to actuator displacements which are transmitted to the ASM at the telescope pupil. In addition to an overall system loop gain, separate gains are applied to the individual DH modes in the reconstruction; these are obtained from the measured modal closed-loop system response. Measured scale factors for the individual responses of the ASM actuators are also applied to account for variations in the sensitivities of 

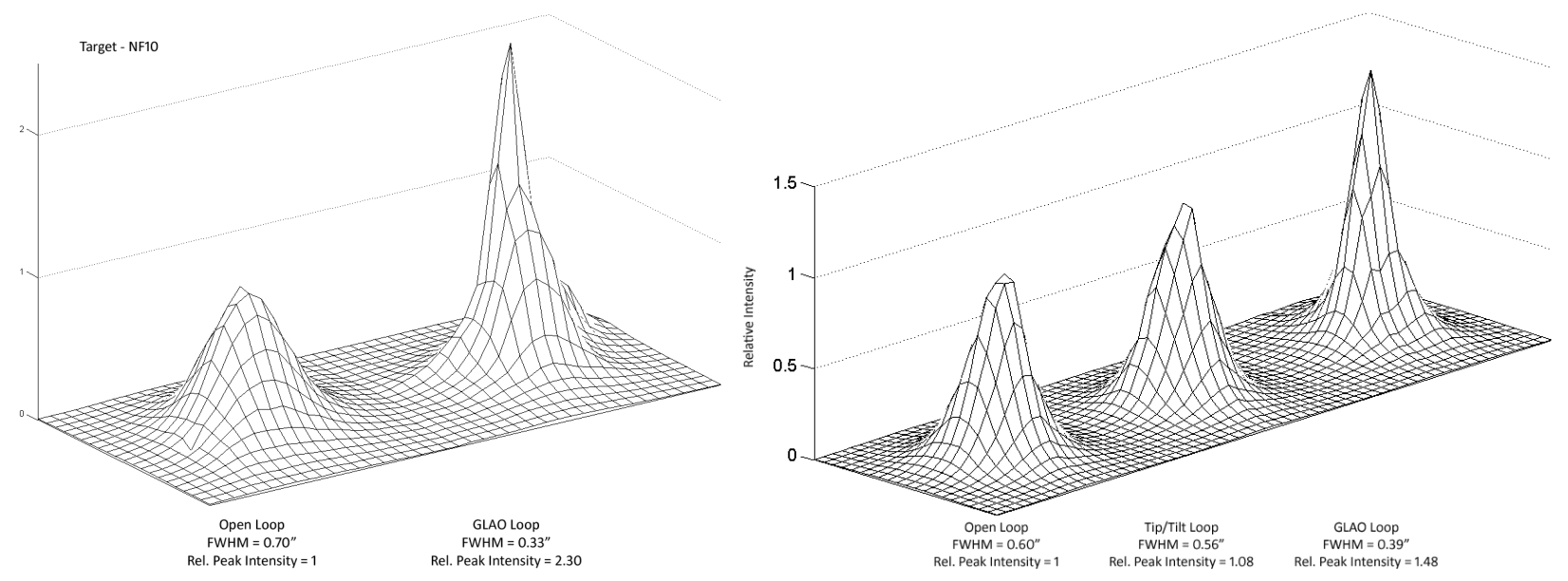

Figure 1. (Left) Comparison of stellar PSFs of target NF10 at $\lambda=2.14 \mu \mathrm{m}$ with no correction and GLAO correction. (Right) Target NF8 at K-short with no correction, tip/tilt only correction, and GLAO correction. Each grid point represents 107 mas.

the associated capacitive sensors. The DH basis functions are used instead of the traditional Zernike polynomials since they provide increased loop stability and a lower RMS error for a given number of controlled modes. Zernike polynomials have large radial derivatives near the edge of the pupil, particularly for high spatial frequency modes. By contrast, the DH functions, with zero radial derivatives at the edge of the pupil, place less stress on actuators at the outer edge of the ASM resulting in lower actuator currents and greater loop stability.

\subsection{First GLAO Results}

Observations were carried out in February 2008 using the MMT multi-laser AO system in order to demonstrate full high order closed loop GLAO correction over a 2 arc minute field. The initial objective was to characterize the performance of the system in open vs. closed loop as well as in closed loop with tip-tilt only correction.

Science images were captured with the instrument PISCES, a near-infrared camera with a 110 arc second field of view and a plate scale of 0.107 arc seconds per pixel. The data consist of sets of $1 \mathrm{~s}$ exposures taken at a rate of approximately 14 exposures per minute using either a standard $\mathrm{K}_{s}$ or $2.14 \mu \mathrm{m}$ narrow band filter. During subsequent analysis, the science exposures are first background subtracted and flat fielded, then de-rotated and co-added to simulate long exposure images.

The first astronomical targets observed on the night of February 19, 2008 were a series of single stars ranging in visual magnitude from 8 to 10 , all with a declination of approximately +40 degrees. The stars were located approximately in the center of the laser beacon constellation with tip/tilt sensing done using the target star itself. Figure 1 shows examples of the stellar point-spread function (PSF) in the seeing limit and with tip/tilt only, and ground layer AO correction.

Target NF10 has a seeing limited full-width at half-maximum (FWHM) of 0.70 arc-seconds, while the closed loop PSF has a FWHM of 0.33 arc-seconds, a reduction of the image width of $53 \%$, with a factor of 2.3 increase in relative peak intensity. The reduction in width represents an improvement in seeing from somewhat worse than median for the site, 65 th percentile, to excellent, at the 15 th percentile. Target NF8 shows only a $10 \%$ decrease in image width with tilt only correction, however during full ground-layer compensation, the FWHM of the image is reduced by $35 \%$, from 0.60 to 0.39 arc-seconds.

Although the results from the February 2008 telescope run demonstrate successful closed loop GLAO operation, the level of correction did not achieve the final expected system performance of 0.1 to 0.2 arc second images in the $\mathrm{K}$ band. This is attributable to two factors. First, due to a weather shortened observing run in February, we did not have time to implement and test the system for measuring and correcting non-common path and other static aberrations. This feature was implemented and initial testing was completed in the subsequent 
May 2008 run. Second, and more seriously, the elevation axis servo control system of the MMT telescope was exhibiting technical problems resulting in the amplification of wind-driven oscillations at $2.3 \mathrm{~Hz}$. In order to maintain stable closed loop GLAO operation, the loop gain had to be significantly reduced, compromising the final level of image correction.

To characterize the performance of the system with field angle, observations were made of the central 2 arc minutes of the globular cluster M13. These data suffer from a static aberration on the ASM to which the LGS WFS is blind. The error is induced by wind and is much more severe in the cluster images than in the single star results of Figure 1. In the image of Figure 2, the effect of the static aberration has been removed post facto. Using an iterative technique, the static wavefront error in the pupil of the telescope has been estimated from the 60 individual $1 \mathrm{~s}$ exposures, and the corresponding PSF removed by deconvolution. The resulting stellar images have a mean FWHM across the field of 0.30 arc seconds with very little variation.

\subsection{Wide-field Performance}

Additional observations were carried out in May 2008 in order to implement and test the high-order NGS WFS in an automated static aberration correction system and to continue to characterize the uniformity of the GLAO corrected PSF over the 2 arc minute wide field image.

The use of a low bandwidth NGS WFS (LBWFS), to correct for quasi-static aberrations in an LGS WFS, was pioneered at Keck. ${ }^{22}$ The MMT Rayleigh LGS system does not suffer from range uncertainty as do sodium laser beacons; nevertheless it does suffer from quasi-static aberrations from a variety of sources. The laser dynamic refocus optics and the periscope assembly which combines the light from all five laser beacons onto a single CCD induce non-common path aberrations that change the location of the LGS spot centroids. Furthermore, the quad cell center of mass calculation used to compute LGS spot centroid positions can be biased by several effects. The zero points for the LGS spot centroids are typically not at the center of a quad cell due to the hexapolar geometry of the prism array Hartmann pattern. These zero points can drift due to flexure and temperature changes during long exposures as well as changes in the alignment of the LGS WFS optics. The center of mass calculation can be biased by asymmetry in the LGS spots caused by WFS optical aberrations and errors in the set point of the dynamic refocus range, truncation of LGS spots by the finite extent of the quad cell, and intrusion by neighboring LGS spots. Additional quasi-static aberrations can be caused by small variations in the reference flat position of the DM. All of these factors create quasi-static aberrations that can slowly vary during a science exposure.

Automated static aberration correction for the MMT LGS WFS system is achieved by measuring the difference in long term average open loop and LGS closed loop aberrations using the NGS WFS on the tip-tilt star. The change in NGS WFS slopes is reconstructed to produce a Zernike modal estimate of the quasi-static nonatmospheric aberrations. These modal corrections are converted to LGS WFS centroid offsets using the LGS WFS influence matrix. Finally, these offsets are used to shift the zero points of the LGS quad cell center of mass calculation during LGS closed loop operation.

Figure 3 shows the 110 arc second field surrounding a $m_{V}=9$ star (20:01:16.72 +20:41:29.8) consisting of the composite of 17 one second PISCES exposures with the $2.14 \mu \mathrm{m}$ filter. Tip-tilt signals were obtained from the bright star in the center of the field, which also served to sense static aberrations. Image improvement was only modest: the tip-tilt star has a measured FWHM of 0.70 arc seconds in open loop and 0.55 arc seconds in GLAO closed loop, a decrease of only $21 \%$. However, in contrast to the results from the February run in Figure 2 , the corrected PSFs are round, showing the effect of removing the static aberration by reference to the NGS WFS.

These results were severely affected by continuing problems with the MMT telescope elevation axis servo control system which had an even larger impact on this run because of generally higher winds. In order to maintain closed loop GLAO operation, the loop gain had to be further reduced, again compromising the final level of image correction. The MMT observatory expects to be able to correct this problem with the servo controllers shortly.

Figure 4 shows the corrected and uncorrected FWHM for the 12 brightest stars in the 110 arc second field shown in figure 3. The mean seeing limited FWHM is $0.721 \pm 0.014$ arc seconds and the mean GLAO corrected 

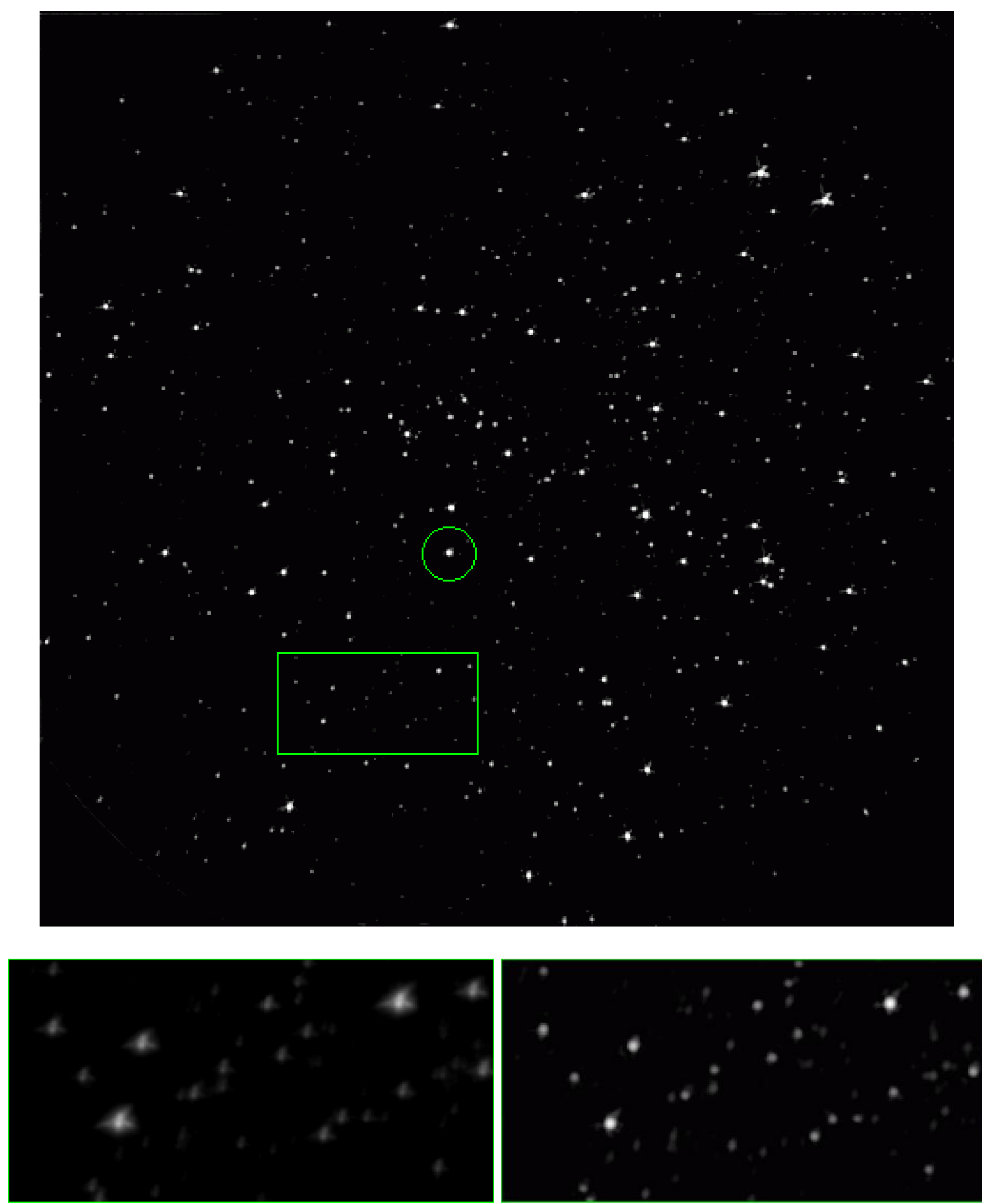

Figure 2. GLAO-corrected image from PISCES of the core of the globular cluster M13. Total exposure time is $60 \mathrm{~s}$, from co-addition of $1 \mathrm{~s}$ exposures in the $\mathrm{K}_{s}$ filter. The tip-tilt star is circled. The static uncorrected pupil-plane aberration has been reconstructed from the image data and its effect removed from this image by deconvolution of the corresponding PSF. The effect can be seen in detail in the two panels below which show a $12 \times 24$ arc second region before (left) and after (right) static aberration removal. 


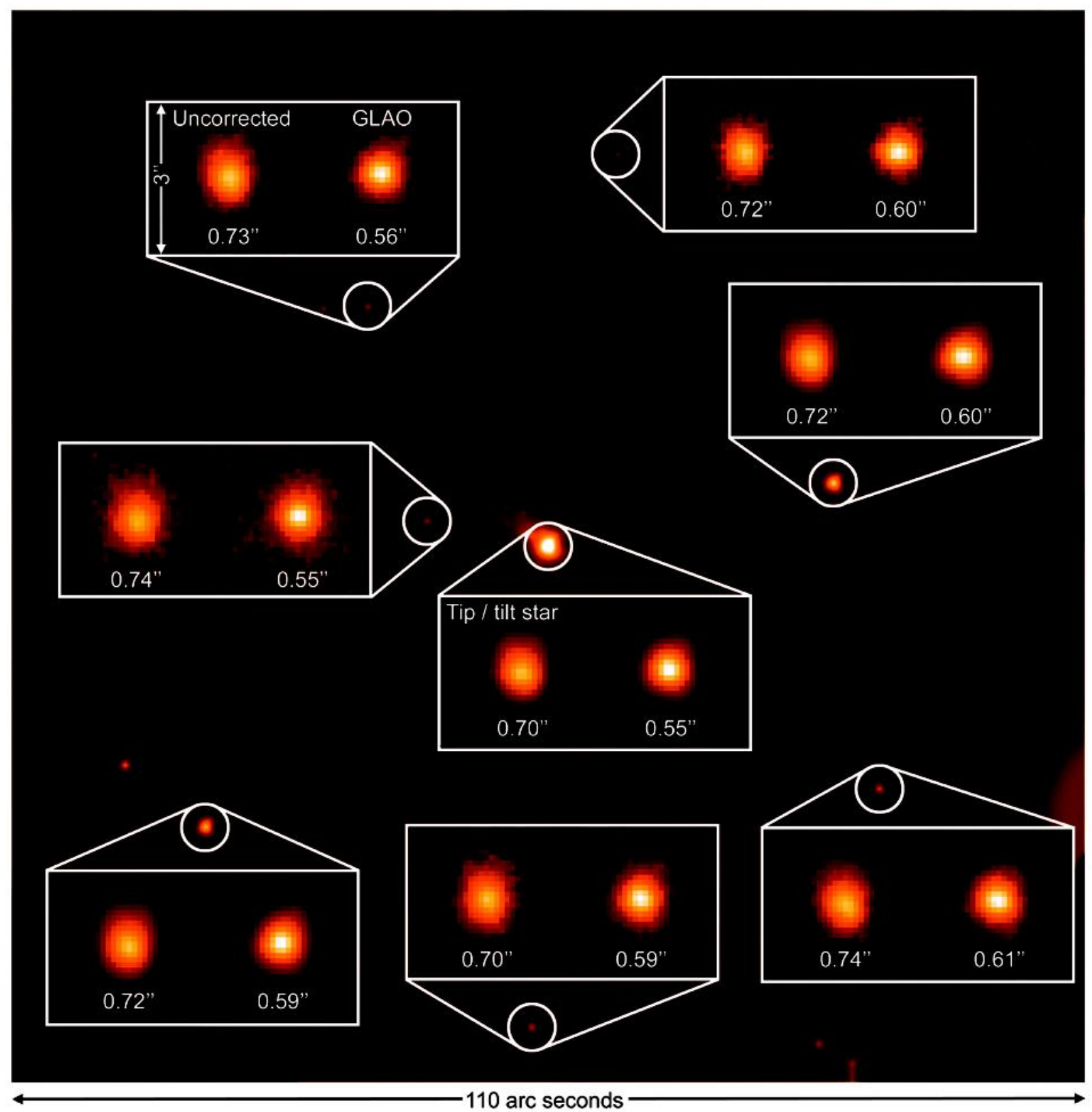

Figure 3. PISCES field showing magnified open and closed loop PSFs. Each breakout box is linearly scaled and normalized to the peak intensity while the background image is logarithmically stretched to more clearly show the location of the faint field stars. The laser guide stars are positioned just outside this 110 arc second field. 


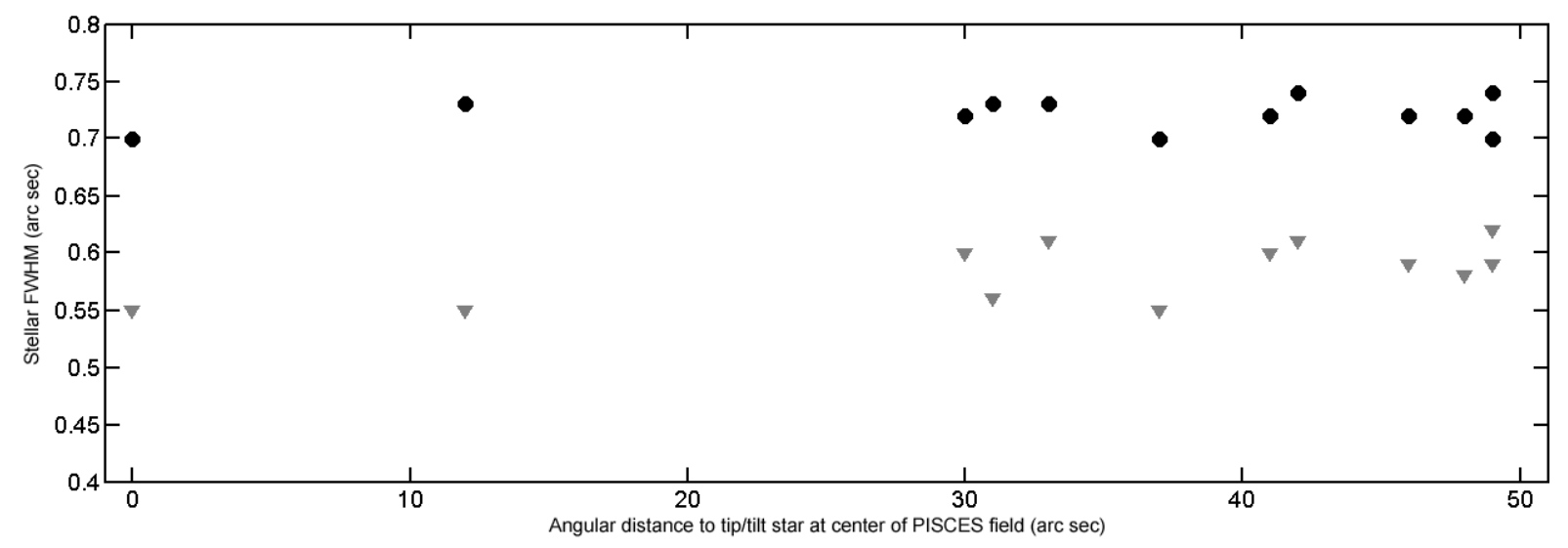

Figure 4. FWHM for the 12 brightest stars in the field shown in figure 3 as a function of their distance from the central tip-tilt star. (Circles) Seeing limited FWHM. (Triangles) GLAO corrected FWHM.

FWHM is $0.584 \pm 0.026$ arc seconds. The GLAO corrected FWHM is fairly constant across the corrected field. These measurements will be repeated once the servo problem is corrected to obtain more definitive results. We expect a substantial overall improvement in the PSF FWHM, though a decrease in closed loop PSF uniformity may be seen due to anisoplanatic effects once the vibration issues have been resolved and we are able to increase the GLAO loop gain.

During these observations, the NGS WFS running at $180 \mathrm{~Hz}$, was passively collecting data in both open and closed loop operation. These data have been analyzed to evaluate the closed loop performance in a manner independent of static aberrations. Table 1 shows the RMS amplitude of Zernike modes, reconstructed from the NGS WFS measurements, grouped by spatial order, along with estimated values for the Fried parameter $r_{0}$.

A reduction in the RMS wavefront error of $42 \%$ had the effect of increasing the observed $r_{0}$ at $\lambda=500 \mathrm{~nm}$ from $\approx 12.3 \mathrm{~cm}$ to $\approx 18.5 \mathrm{~cm}$. This reduction, considering only modes of order 2 through 8 , was $38 \%$ which is consistent with previous open loop studies of GLAO performance at the MMT. ${ }^{7}$ However, the reduction of $43 \%$ for the tip-tilt modes is much lower than the $>80 \%$ correction obtained in previous analysis and is attributable to the low tip-tilt loop gains and the servo oscillations experienced during this run.

Figure 5 shows the open and closed loop amplitude and power spectrum for Zernike mode 2, the tilt mode parallel to the telescope elevation axis as sensed by the fast tip-tilt CCD from a natural star. The power spectrum plot (right) clearly shows that the GLAO control is removing a significant portion of the atmosphere at very low frequencies, below $1.5 \mathrm{~Hz}$. But at the $2.3 \mathrm{~Hz}$ oscillation, the reduction is only a factor of four, which although consistent with the controller operating at low gain is insufficient to recover good image quality. We note that these data were recorded in the most favorable configuration, with the telescope turned at roughly $90^{\circ}$ to the wind and shielded to a large extent by the dome. Pointed into the wind, which at this time was $\approx 50 \mathrm{kph}$, the

\begin{tabular}{lcccccccccccc}
$\begin{array}{l}\text { Correction } \\
\text { method }\end{array}$ & $\begin{array}{c}\text { Set } \\
\text { number }\end{array}$ & $\begin{array}{c}\text { Zernike Order } \\
1\end{array}$ & $\begin{array}{c}2 \\
\mathrm{~nm}\end{array}$ & $\begin{array}{c}\mathrm{nm} \\
\mathrm{nm}\end{array}$ & $\begin{array}{c}4 \\
\mathrm{~nm}\end{array}$ & $\begin{array}{c}5 \\
\mathrm{~nm}\end{array}$ & $\begin{array}{c}6 \\
\mathrm{~nm}\end{array}$ & $\begin{array}{c}7 \\
\mathrm{~nm}\end{array}$ & $\begin{array}{c}8 \\
\mathrm{~nm}\end{array}$ & $\begin{array}{c}1-8 \\
\mathrm{~nm}\end{array}$ & $\begin{array}{c}2-8 \\
\mathrm{~nm}\end{array}$ & $\begin{array}{c}r_{0} \\
\mathrm{~cm}\end{array}$ \\
\hline Uncorrected & 1 & 1137 & 486 & 313 & 230 & 187 & 153 & 131 & 118 & 1330 & 690 & 12.2 \\
& 2 & 1227 & 460 & 307 & 221 & 175 & 145 & 127 & 114 & 1393 & 660 & 12.4 \\
Closed loop & 1 & 671 & 244 & 213 & 145 & 130 & 116 & 115 & 107 & 794 & 425 & 18.2 \\
& 2 & 675 & 235 & 209 & 138 & 125 & 109 & 108 & 100 & 790 & 409 & 18.8 \\
$\begin{array}{l}\text { Mean \% } \\
\text { correction }\end{array}$ & & 43 & 49 & 32 & 37 & 30 & 24 & 14 & 11 & 42 & 38 &
\end{tabular}

Table 1. Comparison of residual wave front error with and without GLAO compensation. 

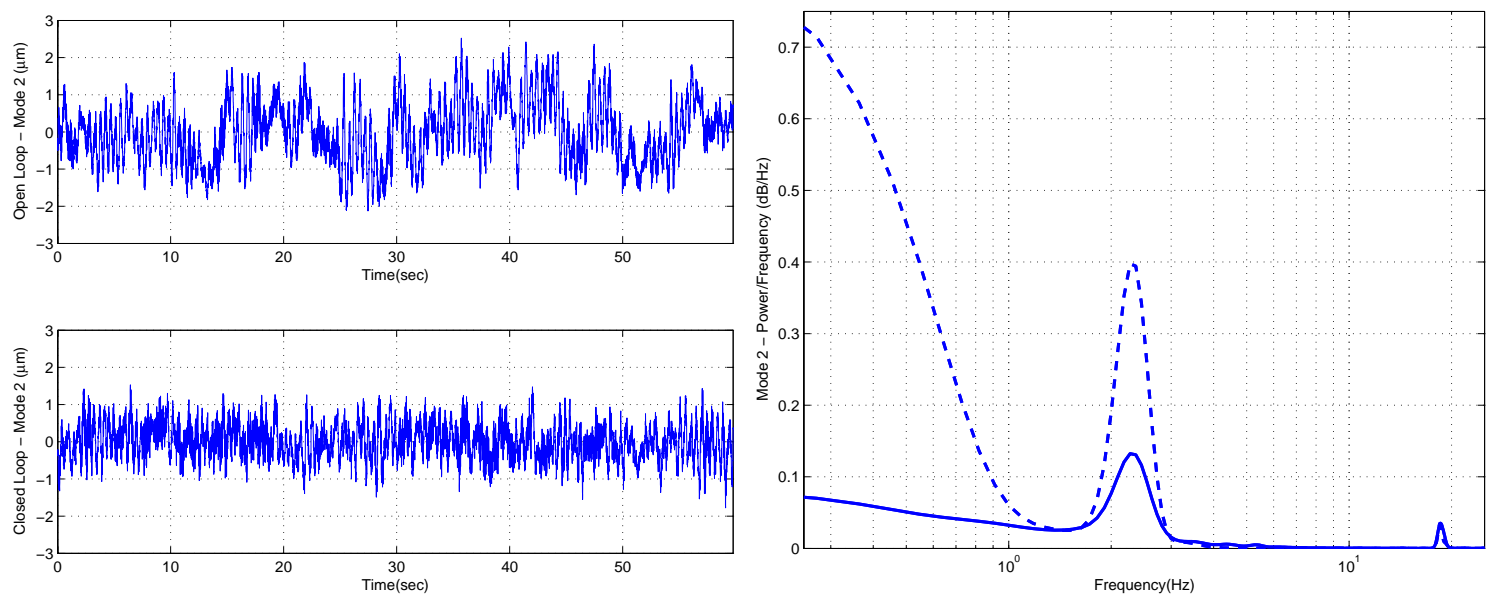

Figure 5. (Left) Open and closed loop modal amplitude for Zernike mode 2 (tip-tilt). (Right) Power spectrum of open (dashed) and closed (solid) loop measurements of Zernike mode 2.

$2.3 \mathrm{~Hz}$ resonance dominated even the lowest frequency atmospheric tilt errors. (The small peak at $\approx 18 \mathrm{~Hz}$ is a vibrational mode of the secondary hub.)

The closed loop response for mode 2 is worse than the open loop response in the region of 3 to $7 \mathrm{~Hz}$. This is due to overshoot from the controller near the limits of its bandwidth, which is about 3 to $4 \mathrm{~Hz}$ at this low gain setting. Once the elevation axis servo oscillation is corrected and the loop gain is increased, the bandwidth of the controller will increase and the overshoot will move out to higher frequencies. We expect the closed loop bandwidth,to improve significantly to $\approx 30 \mathrm{~Hz}$ once the servo issues are addressed.

\section{FUTURE COMMISSIONING WORK}

\subsection{GLAO Shared Risk Science}

Several vibration control issues need to be addressed to improve the system performance. The most serious issue lies with the elevation axis pointing servo currently implemented on the MMT telescope. The closed loop servo response results in gain peaking at $\approx 2.3 \mathrm{~Hz}$ which causes severe stability problems for the AO system. This is particularly evident in high winds. Improvements to the MMT servo controller algorithm, currently being implemented by the MMT observatory, should alleviate gain peaking, allow for higher bandwidth and greater disturbance rejection capability, and result in a more stable LGS system.

The main contribution to the higher order modal RMS residuals, shown in table 1, is the incomplete cancellation of a $14 \mathrm{~Hz}$ rotational mode of the secondary mirror hub. Although the LGS beam projector fast steering mirror has made dramatic reductions in the overall LGS spot motion induced by the rotational mode based on accelerometer signals from the hub, there is still need for further improvement. Optimizing the filtering of the sensed acceleration signals along with increasing the controller update rate will minimize delays, resulting in additional attenuation of the tilt component in the residuals.

There is also a $20 \mathrm{~Hz}$ tilt mode of the hub which affects the residual RMS. A feedforward accelerometer scheme, similar to the $14 \mathrm{~Hz}$ correction, ${ }^{23}$ is being implemented to actively cancel the vibration. In addition, the DM actuator response is currently being refined in a set of calibration measurements on the MMT ASM test stand $^{24}$ in order to incrementally improve performance.

Once these issues are addressed, we expect to return to the MMT telescope in the fall of 2008 for further GLAO commissioning work and to begin shared risk science observations. Detailed PSF morphology will be obtained using Clio, which has a plate scale of 48 mas per pixel. PISCES will again be used to examine the uniformity of the PSF across its 110 arc second field. We will also examine the effect of varying tip-tilt star brightness and field location on the performance of the GLAO correction. 
Shared risk science using GLAO will target two key types of observation: high redshift galaxies and the low end of the stellar initial mass function. ${ }^{25}$ GLAO offers the chance to achieve consistently good imaging quality, well matched to the sizes of high redshift galaxies, in which to integrate down to the required faint flux levels. This is particularly true in the K band, where GLAO should perform well and where HST has little capability. Ground-based K band GLAO imaging will have higher resolution than Spitzer/IRAC, and so will be the reddest band for galaxy morphologies prior to JWST. GLAO observations of Galactic star forming regions in the near IR will generate wide field surveys of sub-stellar objects that will help provide fundamental tests of models for the dynamical evolution of brown dwarfs and stars.

\subsection{LTAO Commissioning}

Laser tomography adaptive optics utilizes the WFS measurements from multiple LGS and tip-tilt measurements from one or more faint natural stars to produce an estimate of the three dimensional distribution of aberrations in the atmosphere. With a single DM, diffraction limited correction can only be achieved in the direction of a single science target which may, however, be located anywhere within the constellation of laser beacons on the sky.

For the MMT multi-laser AO system, the only difference between GLAO and LTAO control of the MMT's adaptive secondary mirror is the reconstructor matrix. Additional inputs characterizing the detailed geometry and atmospheric turbulence profile are necessary to generate an LTAO reconstructor. A wide-field imaging CCD, which has been used for target acquisition in combination with the PISCES science imager, will be used to measure the exact relative positions of the science target and the faint tip-tilt natural star with respect to the locations of the five laser beacons on the sky. In addition, open loop LGS WFS measurements will be used to estimate the $C_{n}^{2}$ profile, ${ }^{26}$ in the direction of the science target, which will yield estimated heights of the atmospheric layers containing significant turbulence strength. This geometric information will be used to build the LTAO reconstructor. ${ }^{27}$

In addition, an upgrade to the LGS WFS instrument at the MMT will provide a pupil imaging mode to more accurately characterize the pupil alignment as well as pupil aberrations and distortions. Since the LTAO reconstructor extrapolates from small differences in the WFS signals from multiple laser beacons, an accurate pupil mapping is critical.

LTAO operation is expected to provide correction qualitatively, and likely also quantitatively, similar to AO systems running with a single sodium LGS, i.e., near full sky coverage at the diffraction limit in $\mathrm{H}$ band and longward.

\section{CONCLUSION}

The multi-LGS program at the MMT is exploring the practical techniques required for wavefront sensing for ground-layer and tomographic correction. Closed loop GLAO operation was successfully demonstrated in February 2008 where wide field, 2 arc minute diameter, image correction reduced stellar image widths by as much as $53 \%$ in the K band. Static aberration correction was tested and additional system characterization was completed in May 2008. Optimization of the system, and the correction of telescope servo control problems, in subsequent commissioning runs in the fall of 2008 will further improve correction performance and is expected to deliver an estimated 0.1 to 0.2 arc second resolution during $75 \%$ of seeing conditions.

Early shared risk scientific programs will focus on seeing improvement with GLAO, taking advantage of existing near infrared instrumentation. The exploitation of routine near infrared seeing of 0.2 arc seconds or better over a field of several arc minutes is likely to be very productive, both for imaging and high resolution multi-object spectroscopy where the many-fold improvement in encircled energy within 0.2 arc seconds will be of particular value.

The implementation of LTAO operation will soon provide near full sky coverage at the diffraction limit. Existing instrumentation at the MMT will be used to perform diffraction limited imaging and spectroscopy from 1 through $10 \mu \mathrm{m}$. 


\section{ACKNOWLEDGMENTS}

Observations reported here were made at the MMT, a joint facility of the University of Arizona and the Smithsonian Institution. This work has been supported by the Air Force Office of Scientific Research under grant F49620-01-1-0383 and the National Science Foundation under grants AST-0138347 and AST-0505369.

\section{REFERENCES}

1. F. Rigaut, "Ground-conjugate wide field adaptive optics for the ELTs," in Beyond Conventional Adaptive Optics, E. Vernet, R. Raggazzoni, S. Esposito, and N. Hubin, eds., Proc. ESO 58, pp. 11-16, 2002.

2. D. Andersen, J. Stoesz, S. Morris, M. Lloyd-Hart, D. Crampton, T. Butterley, B. Ellerbroek, L. Jollissaint, N. M. Milton, R. Myers, K. Szeto, A. Tokovinin, J. P. Veran, and R. Wilson, "Performance modeling of a wide-field ground-layer adaptive optics system," PASP 118, pp. 1574-1590, 2006.

3. M. L. Louarn and N. Hubin, "Improving the seeing with wide-field adaptive optics in the near-infrared," Mon Not R Astron Soc 365, pp. 1324-1332, 2006.

4. A. Tokovinin, "Seeing improvement with ground-layer adaptive optics," PASP 116, pp. 941-951, 2004.

5. M. Lloyd-Hart, C. Baranec, N. M. Milton, T. Stalcup, M. Snyder, and J. R. P. Angel, "First tests of wavefront sensing with a constellation of laser guide beacons," ApJ 634, pp. 679-686, 2005.

6. M. Lloyd-Hart, C. Baranec, N. M. Milton, T. Stalcup, M. Snyder, and J. R. P. Angel, "Experimental results of ground-layer and tomographic wavefront reconstruction from multiple laser guide stars," OptEx 14, pp. 7541-7551, 2006.

7. C. Baranec, M. Lloyd-Hart, N. M. Milton, T. Stalcup, M. Snyder, V. Vaitheeswaran, D. W. McCarthy, and A. R, "Astronomical imaging using ground-layer adaptive optics," in Astronomical Adaptive Optics Systems and Applications III, R. K. Tyson and M. Lloyd-Hart, eds., Proc. SPIE 6691, p. 669120, 2007.

8. E. Marchetti, R. Brast, B. Delabre, R. Donaldson, E. Fredrigo, C. Frank, N. Hubin, J. Kolb, J. Lizon, M. Marchesi, S. Oberti, R. Reiss, J. Santos, C. Soenke, S. Tordo, A. Baruffolo, P. Bagnara, and T. C. consortium, "On-sky Testing of the Multi-Conjugate Adaptive Optics Demonstrator," The Messenger 129, pp. 8-13, 2007.

9. H. Bouy, J. Kolb, E. Marchetti, E. Martin, N. Huelamo, and D. B. y Navascues, "Multi-conjugate adaptive optics images of the Trapezium cluster," Astronomy and Astrophysics 477, pp. 681-690, 2008.

10. C. Benn, R. Rutten, D. Abrams, T. Agocs, D. Cano Infants, T. Gregory, J. Guerra, O. Martin, T. Morris, R. Myers, S. Rix, I. Skillen, J. Skvarc, and S. Tulloch, "GOAS/NAOMI: ground-layer AO at the William Herschel Telescope," in Adaptive Optics Systems, N. N. Hubin, C. E. Max, and P. L. Wizinowich, eds., Proc. SPIE 7015, p. 701574, 2008.

11. A. Tokovinin, N. van der Bliek, R. Cantarutti, M. Martinez, E. Mondaca, A. Montane, P. Schurter, and R. Tighe, "SAM: a facility GLAO instrument," in Adaptive Optics Systems, N. N. Hubin, C. E. Max, and P. L. Wizinowich, eds., Proc. SPIE 7015, p. 7015157, 2008.

12. T. E. Stalcup, Design and construction of a multiple beam laser projector and dynamically refocused wavefront sensor. PhD thesis, University of Arizona, 2006.

13. G. Brusa-Zappellini, A. Riccardi, S. Ragland, S. Esposito, C. D. Vecchio, L. Fini, P. Stefanini, V. Biliotti, P. Ranfagni, P. Salinari, D. Gellieni, R. Biasi, P. Mantegazza, G. Sciocco, G. Noviello, and S. Invernizzi, "Adaptive secondary P30 prototype: laboratory results," in Adaptive Optical System Technologies, D. Bonaccini and R. K. Tyson, eds., Proc. SPIE 3353, pp. 764-775, 1998.

14. F. Wildi, G. Brusa, M. Lloyd-Hart, L. Close, and A. Riccardi, "First light for the 6.5-m MMT adaptive optics system," in Astronomical Adaptive Optical Systems and Applications, R. K. Tyson and M. Lloyd-Hart, eds., Proc. SPIE 5169, pp. 17-25, 2003.

15. C. J. Baranec, Astronomical adaptive optics using multiple laser guide stars. $\mathrm{PhD}$ thesis, University of Arizona, 2007.

16. J. Georges, Dynamically refocused Rayleigh beacons for adaptive optics. PhD thesis, University of Arizona, 2003.

17. D. McCarthy, J. Ge, J. Hinz, R. Finn, and R. de Jong, "PISCES: A wide-field, 1-2.5 micron camera for large-aperture telescopes," PASP 113, pp. 353-361, 2001. 
18. M. Freed, P. Hinz, M. Meyer, N. M. Milton, and M. Lloyd-Hart, "Clio: A 5 micron camera for the detection of giant exoplanets," in Ground-based Instrumentation for Astronomy, A. Moorwood and M. Iye, eds., Proc. SPIE 5492, pp. 1561-1571, 2004.

19. D. McCarthy, J. Burge, R. Angel, J. Ge, R. Sarlot, B. Fitz-Patrick, and J. Hinz, "ARIES: Arizona infrared imager and echelle spectrograph," in Infrared Astronomical Instrumentation, A. Fowler, ed., Proc. SPIE 3354, pp. 750-754, 1998.

20. P. Hinz, R. Angel, N. Woolf, W. Hoffmann, and D. McCarthy, "BLINC: a testbed for nulling interferometry in the thermal infrared," in Interferometry in Optical Astronomy, P. Lena and A. Quirrenbach, eds., Proc. SPIE 4006, pp. 349-353, 2001.

21. N. M. Milton and M. Lloyd-Hart, "Disk harmonic functions for adaptive optics simulations," in Adaptive Optics: Analysis and Methods, B. L. Ellerbroek, ed., Proc. OSA, 2005. Charlotte, North Carolina, June 6-9.

22. M. van Dam, A. Bouchez, D. L. Mignant, E. Johansson, P. Wizinowich, R. Campbell, J. Chin, S. Hartman, R. Lafon, P. Stomski, and D. Summers, "The W. M. Keck observatory laser guide star adaptive optics system: Performance characterization," PASP 118, pp. 1574-1590, 2006.

23. T. E. Stalcup and K. Powell, "Image motion correction using accelerometers at the MMT Observatory," in Advanced Optical and Mechanical Technologies in Telescopes and Instrumentation, E. Atad-Ettedgui and D. Lemke, eds., Proc. SPIE 7018, p. 701854, 2008.

24. T. E. Stalcup, O. F. Durney, and T. Conners, "A test stand for the MMT observatory adaptive secondary," in Adaptive Optics Systems, N. N. Hubin, C. E. Max, and P. L. Wizinowich, eds., Proc. SPIE 7015, p. 7015224, 2008.

25. M. Lloyd-Hart, T. Stalcup, C. Baranec, N. M. Milton, M. Rademacher, M. Snyder, M. Meyer, and D. Eisenstein, "Scientific goals for the MMT's multi-laser-guided adaptive optics," in Advances in Adaptive Optics II, B. Ellerbroek and D. Bonaccini, eds., Proc. SPIE 6272, p. 62724J, 2006.

26. N. M. Milton, M. Lloyd-Hart, J. Bernier, and C. Baranec, "Real-time atmospheric turbulence profile estimation using modal covariance measurements from multiple guide stars," in Astronomical Adaptive Optics Systems and Applications III, R. K. Tyson and M. Lloyd-Hart, eds., Proc. SPIE 6691, p. 66910B, 2007.

27. M. Lloyd-Hart and N. M. Milton, "Fundamental limits on isoplanatic correction with multi-conjugate adaptive optics," JOSA A 20, pp. 1949-1957, 2003. 